\title{
REVIEW ARTICLE Cannabidiol in Medicine: A Review of its Therapeutic Potential in CNS Disorders
}

\author{
Caterina Scuderi $^{1}$, Daniele De Filippis ${ }^{2}+$, Teresa Iuvone $^{2}+$, Angelo Blasio ${ }^{1}$, Antonio Steardo ${ }^{1}$ \\ and Giuseppe Esposito ${ }^{1 *}$ \\ ${ }^{1}$ Department of Physiology and Pharmacology 'V. Erspamer', Sapienza University of Rome, Italy \\ ${ }^{2}$ Department of Experimental Pharmacology, University of Naples 'Federico II', Italy
}

\begin{abstract}
Cannabidiol (CBD) is the main non-psychotropic component of the glandular hairs of Cannabis sativa. It displays a plethora of actions including anticonvulsive, sedative, hypnotic, antipsychotic, antiinflammatory and neuroprotective properties. However, it is well established that CBD produces its biological effects without exerting significant intrinsic activity upon cannabinoid receptors. For this reason, CBD lacks the unwanted psychotropic effects characteristic of marijuana derivatives, so representing one of the bioactive constituents of Cannabis sativa with the highest potential for therapeutic use.

The present review reports the pharmacological profile of CBD and summarizes results from preclinical and clinical studies utilizing CBD, alone or in combination with other phytocannabinoids, for the treatment of a number of CNS disorders. Copyright @ 2008 John Wiley \& Sons, Ltd.
\end{abstract}

Keywords: cannabidiol; Cannabis sativa; CNS disorder; phytocannabinoid.

\section{INTRODUCTION}

Cannabidiol (CBD) is the main non-psychotropic component of the glandular hairs of Cannabis sativa and belongs to the so-called 'cannabinoids' (CBs). This term refers to a heterogeneous family of molecules usually exhibiting pharmacological properties by interacting with specific receptors. So far, two membrane receptors for CBs, both coupled to $\mathrm{G}$ protein and named CB1 and $\mathrm{CB} 2$, have been identified. While CB1 receptors are mainly expressed in the central and the peripheral nervous systems, $\mathrm{CB} 2$ receptors have been reported to be more abundantly detected in cells of the immune system (for review see Pertwee, 2006). Moreover, two orphan G protein-coupled receptors, GPR119 and GPR55, possibly activated by multiple different cannabinoid ligands (Brown, 2007), have been recently proposed as novel cannabinoid receptors. CBs can be separated into three different groups: endogenous (endocannabinoids), synthetic and phytocannabinoids. The latter group includes terpenophenolic substances extracted from Cannabis sativa, such as $\Delta 9$-tetrahydrocannabinol ( $\triangle 9$-THC) and CBD. Cannabinoid pharmacology is a field rapidly expanding and the therapeutic properties of cannabinoid receptor agonists include analgesia, muscle relaxation, immunosuppressant, antiinflammatory and antiallergic effects, improvement of mood, stimulation of appetite, antiemesis, lowering of intraocular pressure, bronchodilatation, neuroprotection and antineoplastic effects (Pertwee, 2005). Despite the emerging evidence regard-

* Correspondence to: Dr Giuseppe Esposito, Department of Physiology and Pharmacology, 'V. Erspamer', Sapienza University of Rome, P. le A. Moro, 5-00185 Rome, Italy.

E-mail: esposgiu@unina.it

${ }^{\dagger}$ Endocannabinoid Research Group. ing therapeutic activities of $\mathrm{CBs}$, their effective introduction in clinical use is still controversial and strongly limited by the unavoidable psychotropic effects exhibited by many of them. In this context, much attention has been focused on CBD that constitutes up to $40 \%$ of the Cannabis extract, and represents one of the most promising candidates for clinical utilization due to its remarkable lack of any cognitive and psychoactive actions. Finally, CBD is regarded as an interesting putative lead compound to develop cannabinomimetic drugs, because of its excellent tolerability in humans (for review see Mechoulam and Hanus, 2002).

\section{CBD PHARMACOLOGY}

Cannabidiol was isolated from marijuana in the late 1930s, but only in the 1963 were its structure and stereochemistry first elucidated (Mechoulam and Shvo, 1963). Early studies focusing on CBD pharmacology started in the 1970s, with the first relevant finding concerning its hypnotic and anticonvulsant properties, published in 1981 (Carlini and Cunha, 1981). Since then, a large body of pharmacological effects has been demonstrated, both in preclinical and in clinical studies.

Up to the present, the molecular pharmacology of CBD has not yet been well defined and little is known about a possible CBD-receptor-mediated signalling pathway. Since it was previously demonstrated that CBD binds with a low affinity to both $\mathrm{CB} 1$ and $\mathrm{CB} 2$ cannabinoid receptors, much research was aimed at recognizing CB1 and CB2 independent modes of action for this phytocannabinoid. To date, different molecular targets have been proposed. The first evidence that CBD can bind to sites different from cannabinoid receptors was offered by the observation that natural CBD and the (+)-synthetic 
one both stimulate the type-1 vanilloid receptor (Bisogno et al., 2001). Other studies indicated that CBD also binds to $5-\mathrm{HT}_{1 \mathrm{~A}}$ and such an interaction was suggested to account for the attenuation of cerebral infarction size occurring during ischemia (Mishima et al., 2005) and also for its anxiolytic effect (Campos and Guimarães, 2008). It has been also reported that CBD may behave as an allosteric modulator at $\mu$ and $\delta$ opioid receptors, even if the effects occur at very high levels of phytocannabinoid, so that this modulation cannot be expected to contribute markedly to the CBD actions exerted in vivo (Kathmann et al., 2006).

However, although CBD displays very low affinity for both $\mathrm{CB} 1$ and $\mathrm{CB} 2$, it has been reported recently that it can operate as a $\mathrm{CB} 2$ receptor inverse agonist and this may, at least in part, contribute to its widely documented antiinflammatory properties (Thomas et al., 2007).

Furthermore it is commonly recognized that many CBD effects are mainly due to its antioxidant activity as first demonstrated by Hampson et al. (1998) that exposed rat cortical neuron cultures to a toxic level of the excitatory neurotransmitter glutamate. On that occasion, the authors showed that CBD exerted a potent antioxidant activity, resulting in a more protective effect than either ascorbate or $\alpha$-tocopherol, against glutamate-mediated neurotoxicity.

\section{CBD PHARMACOKINETICS}

The correct use of CBD in human therapy necessarily requires basic information related to pharmacokinetics. Cannabis derivatives are usually inhaled or orally administered. Other routes, including rectal, transdermic, eye drops, aerosols and intravenous have been used in a small number of studies so that the relevance of findings is limited. Recently, it was demonstrated in rabbits that sublingual administration of a solid CBD/beta-cyclodextrin complex may provide an alternative formulation for sublingual administration (Mannila et al., 2007). The pharmacokinetics of CBD is quite complicated and in many aspects resembles that of $\triangle 9$-THC. Once taken orally, CBD bioavailability ranges between 13\% and 19\%, due to a marked first-pass effect, while the systemic bioavailability of inhaled CBD in a group of cannabis users was $31 \%$ (range $11-45 \%$ ). The plasma pattern was similar to that of $\Delta 9$-THC. Daily oral doses of CBD $10 \mathrm{mg} / \mathrm{kg} /$ day chronically administered resulted in mean plasma concentrations of 5.9-11.2 ng/mL (Consroe et al., 1991a). CBD is rapidly distributed when intravenously administered, and easily passes the blood-brain barrier. CBD shows a prolonged elimination; its terminal halflife is about $9 \mathrm{~h}$, and it is excreted preferentially in the urine, both free and as its glucuronide compound (Samara et al., 1990).

Cannabidiol impairs hepatic drug metabolism in several animal species, and inhibits mouse hepatic metabolism through the inactivation of specific cytochrome P450 belonging to the $2 \mathrm{C}$ and $3 \mathrm{~A}$ subfamilies (Bornheim et al., 1993). The metabolism of CBD showed biotransformation routes typically observed for cannabinoids (Harvey et al., 1991a). It undergoes multiple hydroxylations, oxidations to carboxylic acids, beta-oxidation, conjugation and epoxidation (Harvey et al., 1991a). Conjugation with fatty acids, first observed with $\Delta 9$ - and $\Delta 8$-THC, provides a potent means of increasing the lipophilicity and, hence, tissue accumulation (Leighty et al., 1976). CBD-7-oic acid together with CBD-glucuronide represent the most abundant products of CBD metabolism detected in human urine (Harvey et al., 1991b). Unlike $\Delta 9$-THC a remarkable percentage of unchanged CBD is excreted in the faeces (Wall et al., 1976).

\section{CBD TOXICITY}

Cannabidiol exhibits very low toxicity in humans and in other species: the $\mathrm{LD}_{50}$ after intravenous administration to rhesus monkeys was $212 \mathrm{mg} / \mathrm{kg}$ (Rosenkrantz et al., 1981). The oral $\mathrm{LD}_{50}$ has not been established, but in 1981 Rosenkrantz showed that an oral dose of CBD 20-50 times larger than the intravenous route is required to initiate severe intoxication (Rosenkrantz et al., 1981). CBD does not cause relevant CNS alterations. Moreover, a large body of studies failed to reveal teratogenic or mutagenic effects induced by CBD (Dalterio et al., 1984; Matsuyama and Fu, 1981).

\section{PREPARATION CONTAINING CBD}

Because of its very low toxicity in humans, a huge number of trials have been performed to assess the clinical efficacy of CBD in different pathologies. Starting from 2001, most of the trials have been carried out utilizing Sativex ${ }^{\circledR}$, the only commercially available preparation containing $\mathrm{CBD} / \triangle 9$-THC. At the moment, four different formulations of Sativex ${ }^{\circledR}$ are under investigation, including the high THC extract (Tetranabinex ${ }^{\circledR}$ ), THC:CBD (narrow ratio), THC:CBD (broad ratio) and the high $\mathrm{CBD}$ extract $\left(\right.$ Nabidiolex $\left.^{\circledR}\right)$ (No authors listed, 2003). Three Sativex ${ }^{\circledR}$ delivery systems exist: the oromucosal spray, sublingual tablets and inhaled (but not smoked) dosage forms.

In 2005 the oromucosal spray administration of Sativex ${ }^{\circledR}$ was approved for the treatment of multiple sclerosis symptoms (Perras, 2005).

It has been proved that co-administration of CBD and $\triangle 9$-THC may alter the pharmacological effect of the latter, potentiating some putative benefits, while attenuating some of its negative effects (Karniol et al., 1974; Zuardi et al., 1982; Russo and Guy, 2006).

\section{CBD IN SOME CNS DISORDERS}

The neuroprotective actions of $\mathrm{CBD}$, mainly due to its antiinflammatory and antioxidant properties, have been well documented (Hampson et al., 1998). Recently, a neuroprotective mechanism of $\mathrm{CBD}$ has also been confirmed in a mouse model of ischemia, where CBD explicates the cerebroprotective action via a cannabinoid receptor-independent myeloperoxidase-inhibiting mechanism (Hayakawa et al., 2007), in addition to a $5 \mathrm{HT}_{1 \mathrm{~A}}$ receptor action (Mishima et al., 2005). It has also been demonstrated that CBD reverses binge ethanol-induced neurotoxicity, once again, via a cannabinoid receptorindependent antioxidant mechanism (Hamelink et al., 
2005). The potential of CBD to attenuate the excessive formation of peroxynitrites induced by glutamate also contributes to its neuroprotective effects, as demonstrated by the in vitro results pointing to the ability of CBD to induce the prevention of retinal apoptosis (El-Remessy et al., 2003). These early data, together with the lack of psychotropic effects and the low toxicity, supports the notion that $\mathrm{CBD}$ may be considered a potentially useful therapeutic agent for the treatment of a number of neurological disorders.

\section{Epilepsy}

Work carried out in the early 1970s demonstrated that CBD possesses a rather potent protective effect against convulsions in a variety of animal models of epilepsy (Carlini et al., 1973; Karler et al., 1973; Karler and Turkanis, 1980; Consroe and Wolkin, 1977a, 1977b). Indeed, some preclinical evidence showed that CBD was remarkably effective against focal seizures, including temporal lobe epilepsy and generalized convulsion induced by both electroshock and GABA receptor blockers. In these experimental paradigms of epilepsy the effects of CBD were considered almost equivalent to those exerted by antiepileptic compounds commonly used in human therapy. Interestingly, CBD was observed to potentiate the anticonvulsant activity of barbiturates and diphenylhydantoin. In this respect it is worth noting that phenytoin and CBD both display similar stereochemical requirements for anticonvulsant drug action (Tamir et al., 1980). Based on these findings, CBD is regarded, among CBs, as the most promising candidate for antiepileptic therapy because of its powerful anticonvulsant properties in the absence of any relevant toxicity. However, besides a great deal of preclinical results, the available literature on CBD use as an anticonvulsant in human therapy is still limited, because it concerns a very few patients.

Many anecdotal reports have confirmed that CBD exerts considerable anticonvulsant activity and the results are efficacious in protecting against partial and tonic-clonic generalized seizures. Indeed, it has been reported that in epileptics who smoked cannabis to control seizures, interrupting cannabis use induced the reappearance of convulsions, whereas reverting to consumption limited symptoms.

Only one double-blind controlled trial was performed to explore the anticonvulsant activity of CBD in 15 patients suffering from frequent attacks of 'secondarily generalized epilepsy' with temporal focus, unresponsive to standard treatment (Cunha et al., 1980). The results of this study indicated that $50 \%$ of patients taking CBD remained virtually symptoms-free for the duration of the trial, with no significant toxicity or side effects due to CBD administration. The rest of the patients under medication improved markedly, whereas subjects receiving the placebo remained unchanged. Despite these intriguing results the trial was never continued, in all probability because of the huge amounts of drug required.

Nowadays, CBD treatment has been also proposed as a promising therapeutic tool in monotherapy in children suffering from convulsions, resistant to conventional treatment, even though $\mathrm{CBD}$ was found to be ineffective against absence seizures produced in laboratory animals (Cortesi and Fusar-Poli, 2007). This suggestion has been advanced for the absence of tolerance to anticonvulsant properties, also during prolonged treatment with CBD. This aspect is relevant in subjects who have been treated previously with a high-dose of anticonvulsants or with a combination of them. Moreover, the possibility of administering CBD by metered dose pump aerosol spray guarantees a very useful system of administration in children with a poor compliance.

\section{Psychosis}

Convergent evidence links the habitual use of Cannabis to a risk of developing schizophrenia or schizophrenialike psychosis, especially in vulnerable subjects. This effect has been attributed to $\triangle 9$-THC, the main psychoactive constituent of the plant. Conversely, different preclinical studies have provided evidence for a potential antipsychotic activity of CBD, with a pharmacological profile resembling that of a neuroleptic drug. Indeed, the effects of CBD were similar to those produced by haloperidol in a rodent model predictive of antipsychotic activity, using different doses of drugs (Zuardi et al., 1991). Both CBD and haloperidol reduced the occurrence of apomorphineinduced stereotypies, however, CBD did not provoke catalepsy, even at the highest doses. These results have supported the assumption that CBD possesses antipsychotic properties, without generating extrapyramidal side-effects, thus suggesting the hypothesis that CBD may work as an atypical antipsychotic (Zuardi et al., 1991). According to this view, the pharmacological profile of CBD was reported to be comparable to that exhibited by clozapine. Interestingly both drugs, in contrast to conventional neuroleptics, induce c-fos expression in mesolimbic and mesocortical structures, but not in basal ganglia (Guimarães et al., 2004).

The first available clinical data, related to this pathology, referred to CBD effects observed in a 19year-old black woman with schizophrenia (Zuardi et al., 1995). The administration of CBD resulted in a significant improvement of psychotic symptoms with an efficacy evaluated as equivalent to that of haloperidol. However, a subsequent clinical study aimed at verifying the efficacy of CBD monotherapy in patients with treatment-resistant schizophrenia (TRS) demonstrated only a mild improvement in one of three patients treated with CBD monotherapy, suggesting that such an approach has to be considered unsatisfactory for TRS (Zuardi et al., 2006). On the other hand, findings have been published reinforcing the suggestions of previous work pointing to the antipsychotic properties of CBD. Indeed the results have demonstrated that smoking some strains of Cannabis containing more CBD, in addition to $\triangle 9$-THC, may be protective against psychotic-like symptoms induced by $\Delta 9$-THC alone (Morgan and Curran, 2008).

\section{Anxiety}

The results from several studies performed in the 1980s suggested that CBD displays sedative and antianxiety properties (Pickens, 1981; Musty, 1984; Musty et al., 1984; Zuardi et al., 1982). Such evidence prompted further investigations to be carried out, employing different paradigms, aimed at better defining its intrinsic anxiolytic efficacy. The findings confirmed that CBD 
exerts significant anxiolytic activity and that antianxiety effects may exhibit an inverted U-shaped dose-response curve in rodents, with the higher doses being no longer effective (Guimaraes et al., 1990, 1994). Moreover, using the elevated plus maze, anxiolytic effects were also reported for the three CBD derivates: HU219, HU252, HU-291 (Guimaraes et al., 1994). The mechanism by which CBD exerts its anxiolytic effects still remains not fully clarified. However, using a conditioned emotional paradigm, the Vogel conflict test, a model of anxiety based on the conflict between drinking and punishment, it was proved that CBD-mediated anxiolytic activity is independent of benzodiazepine receptors or of non-specific drug interference on the nociceptive threshold or water consumption (Moreira et al., 2006). Recently, the binding at $5 \mathrm{HT}_{1 \mathrm{~A}}$ receptor has been considered as a possible molecular target for its antianxiety activity. Indeed, evidence has been provided suggesting that activation of $5 \mathrm{HT}_{1 \mathrm{~A}}$ receptors located in the dorsolateral periacqueductal gray could be one of the mechanisms of the anxiolytic effect observed with this compound after systemic administration (Campos and Guimarães, 2008).

In contrast to preclinical studies, few human trials have been conducted to evaluate the efficacy of CBD as an antianxiety agent. Oral administration of CBD in healthy volunteers was found to reduce the anxiogenic effects of $\Delta 9$-THC (Zuardi et al., 1982). This property does not seem to implicate pharmacokinetic interactions with $\Delta 9$ THC, suggesting that CBD possesses intrinsic anxiolytic properties. Moreover, the anxiolytic effects of CBD were also observed in healthy volunteers submitted to a simulation of a public speaking test. On that occasion CBD (300 mg/per os) exhibited an efficacy comparable to that displayed by diazepam (10 mg/per os) and ipsapirone (5 mg/per os) (Zuardi et al., 1993). Recently, in view of its anxiolytic effect, neuroimaging studies were performed to test whether CBD was able to affect neural functioning in brain regions usually implicated in the pathophysiology of anxiety. The findings demonstrated that CBD modulates neuronal activity in limbic and paralimbic areas, including orbitofrontal, cingulate and medial temporal cortex, and the insula (Crippa et al., 2004).

In addition to anxiolytic actions, early investigations also demonstrated that CBD induces biphasic hypnotic effects in rats as well as enhances sleeping time, compared with placebo, in insomniacs (Monti, 1977; Carlini and Cunha, 1981). However, more recent results from animal studies rendered controversial previous data, since i.c.v. injections of CBD increased the extracellular level of dopamine in rat nucleus accumbens, suggesting that CBD can improve alertness, and might be regarded, on the contrary, as a potential therapeutic tool in sleep disorders such as hypersomnias (Murillo-Rodríguez et al., 2006).

\section{CBD and control of movement disorders}

Anecdotal evidence has suggested a potential beneficial role of CBD, alone or in combination with $\triangle 9-\mathrm{THC}$, in different neurodegenerative diseases, including Parkinson's disease (PD) and Huntington's disease (HD), two chronic illnesses due to degenerative processes involving specific nuclei of the basal ganglia, resulting in the abnormal regulation of movements. Both disorders have been scantly investigated from the clinical point of view, whereas, at the preclinical level, the accumulated findings appear more exhaustive and convincing for the possible medical utilization of CBD to improve symptoms and/ or delay disease progression. According to recent preclinical findings, plant-derived cannabinoids were able to prevent neuronal damage induced by 6-hydroxydopamine unilateral injection into the nigra, pars compacta (LastresBecker et al., 2005). This effect appeared not to implicate $\mathrm{CB}$ receptor mediation while, more likely, it might be due to antioxidant activity, possibly combined with the capability to modulate glial responses, relevant to neural survival. In rats with hemiparkinsonism, generated by the nigral administration of 6-hydroxydopamine, the neuroprotective effects exerted by CBD reverted dopaminergic transmission impairment, by reducing dopaminergic cell death, rather than by enhancing the functional turnover of the surviving neurons (LastresBecker et al., 2005). Early human reports demonstrated a dose-related improvement (ranging from 20\% to 50\%) in parkinsonian patients treated with oral doses of CBD (100-600 mg/day over a 6 week period) (Consroe et al., 1986). Conversely, in a more recent controlled trial of a mixture of $\Delta 9$-THC/CBD (2.5 mg/1.25 mg per capsule), it failed to exert any beneficial effect either on parkinsonism or levodopa-induced dyskinesias (Caroll et al., 2004). Unfortunately no subsequent trials were performed in an attempt to clarify such conflicting results. Certainly, in comparison with the relevance of preclinical data, the limited clinical evidence suggests that human studies should be carried out to verify for good the potential for a future clinical application of CBD in PD.

Similarly, based on anecdotal accounts and the results of preliminary clinical observations CBD was considered to be a compound with therapeutic potential also against hyperkinetic disorders. Indeed, CBD was demonstrated to mitigate apomorphine-induced turning behaviour in 6-hydroxydopamine injected rats, an animal model of hyperkinetic movement disorders, while, on the other hand, it was able to potentiate hypokinesia generated by tetrabenazine (Consroe et al., 1988). More recently CBD was found to protect striatal neurons against the in vivo toxicity of 3-nitropropionic acid, a mitochondrial toxin that replicates some biochemical alterations occurring in HD (Sagredo et al., 2007).

Cannabidiol was investigated for its efficacy in HD, alone or as an add-on drug to the approved therapy with neuroleptics (Consroe et al., 1991b). CBD, at an average daily dose of $10 \mathrm{mg} / \mathrm{kg} / \mathrm{day}$ for 6 weeks, was neither symptomatically effective nor toxic compared with placebo, in neuroleptic-free patients with HD. Considering the negative results on both the therapeutic and the safety measures, there is a question about the dose as well as the duration of the trial. Since these results cannot be considered conclusive, further trials, utilizing CBD alone or in combination with $\triangle 9-\mathrm{THC}$, should be performed to evaluate clinically the real antihyperkinetic value of these molecules.

Amyotrophic lateral sclerosis (ALS) is a fatal neurodegenerative disease characterized by selective loss involving motoneuronal cells in the cortex, brainstem and spinal cord. Recent reports confirm that in the pathophysiology of ALS neuroinflammation and oxidative stress play a crucial role (Turner and Talbot, 2008). Based on this evidence, it is possible to speculate that $\mathrm{CBD}$, because of its antinflammatory and 
antioxidative properties, could be a promising option to improve disturbances and prolong survival in ALS patients. This is strongly reinforced by the observations that $\Delta 9$-THC was able to delay impairment and prolong survival in a mouse transgenic model of ALS, and that similar results were achieved when cannabinol was used (Weydt et al., 2005). Furthermore, these findings have to be considered together with anecdotal reports that recreational smoking of marijuana does improve symptomatology in ALS subjects.

\section{CBD and experimental model of Alzheimer diseases}

To date very promising results have been achieved in the control of $\beta$-amyloid ( $\mathrm{A} \beta$ ) toxicity by utilizing CBD. Although at present it is still not fully clear the precise biochemical mechanisms by which $A \beta$ exerts its detrimental effects, nevertheless its role in inducing neuronal damage and in mediating neuroinflammation. Following this idea, it has been demonstrated that CBD protected differentiated PC12 neuronal cells from $\mathrm{A} \beta$ exposure, through a combination of antioxidant, antiinflammatory and antiapoptotic effects (Iuvone et al., 2004). The CBD antioxidant effect accounts mainly for the survival of cultured neurons, with a potency higher than $\alpha$-tocopherol. Moreover it helps to attenuate $\mathrm{A} \beta$ neurotoxicity, even with a mechanism not possessed by classical antioxidants. In fact, CBD administration results in a blunting of $\mathrm{A} \beta$ induced GSK-3 $\beta$ activation, the key enzyme of the WNT/ $\beta$-catenin pathway, so preventing the hyperphosphorylation of tau proteins and, consequently, neurofibrillary tangle formation.

Cannabidiol decreases the phosphorylation of the stressactivated protein kinase, P38 MAPK, so preventing translocation to the nucleus of $\mathrm{NF}-\kappa \mathrm{B}$ and the subsequent transcription of important pro-inflammatory genes, including that encoding for iNOS protein (Esposito et al., 2006). The CBD antiinflammatory properties were also confirmed in an in vivo study on mice inoculated with $\mathrm{A} \beta$, where $\mathrm{CBD}$ dose-dependently inhibited reactive gliosis, by impairing glial cell activation and proinflammatory mediator release (Esposito et al., 2007).

Despite these encouraging preclinical results, the therapeutic potential of $\mathrm{CBD}$ in $\mathrm{AD}$ is already being investigated. Clinical trials to explore the beneficial effects of $\triangle 9-\mathrm{THC} / \mathrm{CBD}$ mixture in $\mathrm{AD}$ patients are presently in progress.

\section{CONCLUSION}

Cannabidiol exhibits an impressive plethora of actions, including anticonvulsive, sedative, hypnotic, antipsychotic, antiinflammatory and neuroprotective properties. Many of which may be of therapeutic relevance as well as serving as leads for pharmaceutical development. CBD is a compound well tolerated in humans, with a profile of very low toxicity, and devoid of psychoactive and cognitive effects. Preliminary studies highlight these remarkably important properties and encourage further experiments to be performed in more complex systems in order to clarify the mechanism(s) responsible for its molecular and cellular actions. At the same time, since most of the investigations suggesting CBD as a novel medicine with substantial neuroprotective potential have been carried out in the most part in animal or then cellular models, then more clinical trials able to validate its beneficial properties are warranted. The results generated by these trials might allow that the current promising expectatives can progress from the present preclinical evidence to a practical therapeutic application.

\section{REFERENCES}

Bisogno T, Hanus L, De Petrocellis L et al. 2001. Molecular targets for cannabidiol and its synthetic analogues: effect on vanilloid VR1 receptors and on the cellular uptake and enzymatic hydrolysis of anandamide. Br J Pharmacol 134: 845-852.

Bornheim LM, Everhart ET, Li J, Correia MA. 1993. Characterization of cannabidiol-mediated cytochrome P450 inactivation. Biochem Pharmacol 45: 1323-1331.

Brown AJ. 2007. Novel cannabinoid receptors. Br J Pharmacol 152: $567-575$.

Campos AC, Guimarães FS. 2008. Involvement of 5HT1A receptors in the anxiolytic-like effects of cannabidiol injected into the dorsolateral periaqueductal gray of rats. Psychopharmacology (Berl) May 1. [Epub ahead of print].

Carlini EA, Cunha JM. 1981. Hypnotic and antiepileptic effects of cannabidiol. J Clin Pharmacol 21: 417S-427S.

Carlini EA, Leite JR, Tannhauser M, Berardi AC. 1973. Letter: Cannabidiol and Cannabis sativa extract protect mice and rats against convulsive agents. J Pharm Pharmacol 25: 664-665.

Carroll CB, Bain PG, Teare L et al. 2004. Cannabis for dyskinesia in Parkinson disease: a randomized double-blind crossover study. Neurology 63: 1245-1250.

Consroe P, Kennedy K, Schram K. 1991a. Assay of plasma cannabidiol by capillary gas chromatography/ion trap mass spectroscopy following high-dose repeated daily oral administration in humans. Pharmacol Biochem Behav 40: 517-522.

Consroe P, Laguna J, Allender J et al. 1991b. Controlled clinical trial of cannabidiol in Huntington's disease. Pharmacol Biochem Behav 40: 701-708.
Consroe, P, Musty R, Conti L. 1988. Effects of cannabidiol in animal models of neurologic dysfunction. In Marijuana: An International Research Report. National Campaign Against Drug Abuse Monograph, Series no 7, Chesher G, Consroe $\mathrm{P}$, Musty R (eds). Australian Government Publishing Service: Canberra, 147-152.

Consroe P, Sandyk R, Snider SR. 1986. Open label evaluation of cannabidiol in dystonic movement disorders. Int $J$ Neurosci 30: 277-282.

Consroe P, Wolkin A. 1977a. Cannabidiol - antiepileptic drug comparisons and interactions in experimentally induced seizures in rats. J Pharmacol Exp Ther 201: 26-32.

Consroe PF, Wolkin AL. 1977b. Anticonvulsant interaction of cannabidiol and ethosuximide in rats. J Pharm Pharmacol 29: $500-501$.

Cortesi M, Fusar-Poli P. 2007. Potential therapeutical effects of cannabidiol in children with pharmacoresistant epilepsy. Med Hypotheses 68: 920-921.

Crippa JA, Zuardi AW, Garrido GE et al. 2004. Effects of cannabidiol (CBD) on regional cerebral blood flow. Neuropsychopharmacology 29: 417-426.

Cunha JM, Carlini EA, Pereira AE et al. 1980. Chronic administration of cannabidiol to healthy volunteers and epileptic patients. Pharmacology 21: 175-185.

Dalterio S, Steger R, Mayfield D, Bartke A. 1984. Early cannabinoid exposure influences neuroendocrine and reproductive functions in male mice: I. Prenatal exposure. Pharmacol Biochem Behav 20: 107-113.

El-Remessy AB, Khalil IE, Matragoon S et al. 2003. 
Neuroprotective effect of (-)delta9-tetrahydrocannabinol and cannabidiol in $\mathrm{N}$-methyl-D-aspartate-induced retinal neurotoxicity: involvement of peroxynitrite. Am J Pathol 163: 1997-2008.

Esposito G, De Filippis D, Maiuri MC, De Stefano D, Carnuccio R, luvone T. 2006. Cannabidiol inhibits inducible nitric oxide synthase protein expression and nitric oxide production in beta-amyloid stimulated PC12 neurons through p38 MAP kinase and NF-kappaB involvement. Neurosci Lett 399: 91-95.

Esposito G, Scuderi C, Savani C et al. 2007. Cannabidiol in vivo blunts beta-amyloid induced neuroinflammation by suppressing IL-1beta and iNOS expression. Br J Pharmacol 151: 1272-1279.

Guimaraes FS, Chiaretti TM, Graeff FG, Zuardi AW. 1990. Antianxiety effect of cannabidiol in the elevated plus-maze. Psychopharmacology 100: 558-559.

Guimaraes FS, de Aguiar JC, Mechoulam R, Breuer A. 1994. Anxiolytic effect of cannabidiol derivatives in the elevated plus-maze. Gen Pharmacol 25: 161-164.

Guimarães VM, Zuardi AW, Del Bel EA, Guimarães FS. 2004. Cannabidiol increases Fos expression in the nucleus accumbens but not in the dorsal striatum. Life Sci 75: 633-638.

Hamelink C, Hampson A, Wink DA, Eiden LE, Eskay RL. 2005. Comparison of cannabidiol, antioxidants and diuretics in reversing binge ethanol-induced neurotoxicity. J Pharmacol Exp Ther 314: 780-788.

Hampson AJ, Grimaldi M, Axelrod J, Wink D. 1998. Cannabidiol and (-)delta9-tetrahydrocannabinol are neuroprotective antioxidants. Proc Natl Acad Sci USA 95: 8268-8273.

Harvey DJ, Samara E, Mechoulam R. 1991a. Comparative metabolism of cannabidiol in dog, rat and man. Pharmacol Biochem Behav 40: 523-532.

Harvey DJ, Samara E, Mechoulam R. 1991b. Urinary metabolites of cannabidiol in dog, rat and man and their identification by gas chromatography-mass spectrometry. J Chromatogr 562: 299-322.

Hayakawa K, Mishima K, Nozako M et al. 2007. Repeated treatment with cannabidiol but not delta9-tetrahydrocannabinol has a neuroprotective effect without the development of tolerance. Neuropharmacology 52: 1079-1087.

luvone T, Esposito G, Esposito R, Santamaria R, Di Rosa M, Izzo AA. 2004. Neuroprotective effect of cannabidiol, a non-psychoactive component from Cannabis sativa, on betaamyloid-induced toxicity in PC12 cells. J Neurochem 89: 134-141.

Karler R, Cely W, Turkanis SA. 1973. The anticonvulsant activity of cannabidiol and cannabinol. Life Sci 13: 1527-1531.

Karler R, Turkanis SA. 1980. Subacute cannabinoid treatment: anticonvulsant activity and withdrawal excitability in mice. Br J Pharmacol 68: 479-484.

Karler R, Turkanis SA. 1981. The cannabinoids as potential antiepileptics. J Clin Pharmacol 21: 437S-448S.

Karniol IG, Shirakawa I, Kasinski N, Pfeferman A, Carlini EA. 1974. Cannabidiol interferes with the effects of delta 9tetrahydrocannabinol in man. Eur J Pharmacol 28: 172-177.

Kathmann M, Flau K, Redmer A, Tränkle C, Schlicker E. 2006. Cannabidiol is an allosteric modulator at mu- and deltaopioid receptors. Naunyn Schmiedebergs Arch Pharmacol 372: 354-361.

Lastres-Becker I, Molina-Holgado F, Ramos JA, Mechoulam R, Fernández-Ruiz J. 2005. Cannabinoids provide neuroprotection against 6-hydroxydopamine toxicity in vivo and in vitro: relevance to Parkinson's disease. Neurobiol Dis 19: $96-$ 107.

Leighty EG, Fentiman AF Jr, Foltz RL. 1976. Long-retained metabolites of delta9- and delta8-tetrahydrocannabinols identified as novel fatty acid conjugates. Res Commun Chem Pathol Pharmacol 14: 13-28.

Mannila J, Järvinen T, Järvinen K, Jarho P. 2007. Precipitation complexation method produces cannabidiol/beta-cyclodextrin inclusion complex suitable for sublingual administration of cannabidiol. J Pharm Sci 96: 312-319.

Matsuyama SS, Fu TK. 1981. In vivo cytogenetic effects of cannabinoids. J Clin Psychopharmacol 1: 135-140.

Mechoulam R, Hanus L. 2002. Cannabidiol: an overview of some chemical and pharmacological aspects. Part I: chemical aspects. Chem Phys Lipids 121: 35-43.

Mechoulam R, Shvo Y. 1963. Hashish. I. The structure of cannabidiol. Tetrahedron 19: 2073-2078.

Mishima K, Hayakawa K, Abe K et al. 2005. Cannabidiol prevents

Copyright $\odot 2008$ John Wiley \& Sons, Ltd. cerebral infarction via a serotonergic 5-hydroxytryptamine 1A receptor-dependent mechanism. Stroke 36: 1077-1082.

Monti JM. 1977. Hypnotic like effects of cannabidiol in the rat. Psychopharmacology (Berl) 55: 263-265.

Moreira FA, Aguiar DC, Guimarães FS. 2006. Anxiolytic-like effect of cannabidiol in the rat Vogel conflict test. Prog Neuropsychopharmacol Biol Psychiatry 30: 1466-1471.

Morgan CJ, Curran HV. 2008. Effects of cannabidiol on schizophrenia-like symptoms in people who use cannabis. Br J Psychiatry 192: 306-307.

Murillo-Rodríguez E, Millán-Aldaco D, Palomero-Rivero $\mathrm{M}$, Mechoulam R, Drucker-Colín R. 2006. Cannabidiol, a constituent of Cannabis sativa, modulates sleep in rats. FEBS Lett 580: 4337-4345.

Musty R. 1984. Possible anxiolytic effects of cannabidiol. In The Cannabinoids: Chemical, Pharmacological and Therapeutic Aspects, Agurell S, Dewey WL, Willette RE (eds). Academic Press: New York, 795-813.

Musty RE, Conti LH, Mechoulam R. 1984. Anxiolytic properties of cannabidiol. In Marihuana 84: Proceedings of the Oxford Symposium on Cannabis, Harvey DJ (ed.). IRL Press: Oxford, UK, 713-719.

No authors listed. 2003. Cannabis-based medicines - GW pharmaceuticals: high CBD, high THC, medicinal cannabis - GW pharmaceuticals, THC:CBD. Drugs RD 4: 306-309.

Perras C. 2005. Sativex for the management of multiple sclerosis symptoms. Issues Emerg Health Technol 72: 1-4.

Pertwee RG. 2005. Pharmacological actions of cannabinoids. Handb Exp Pharmacol 168: 1-51.

Pertwee RG. 2006. The pharmacology of cannabinoid receptors and their ligands: an overview. Int J Obes (Lond) 30: S13S18.

Pickens JT. 1981. Sedative activity of cannabis in relation to its delta-1-trans-tetrahydrocannabinol and cannabidiol content. Br J Pharmacol 72: 649-656.

Rosenkrantz H, Fleischman RW, Grant RJ. 1981. Toxicity of shortterm administration of cannabinoids to rhesus monkeys. Toxicol Appl Pharmacol 58: 118-131.

Russo E, Guy GW. 2006. A tale of two cannabinoids: the therapeutic rationale for combining tetrahydrocannabinol and cannabidiol. Med Hypotheses 66: 234-246.

Sagredo O, Ramos JA, Decio A, Mechoulam R, Fernández-Ruiz J. 2007. Cannabidiol reduced the striatal atrophy caused 3-nitropropionic acid in vivo by mechanisms independent of the activation of cannabinoid, vanilloid TRPV1 and adenosine A2A receptors. Eur J Neurosci 26: 843-851.

Samara E, Bialer M, Harvey DJ. 1990. Pharmacokinetics of urinary metabolites of cannabidiol in the dog. Biopharm Drug Dispos 11: 785-795.

Tamir I, Mechoulam R, Meyer AY. 1980. Cannabidiol and phenytoin: a structural comparison. J Med Chem 23: 220-223.

Thomas A, Baillie GL, Phillips AM, Razdan RK, Ross RA, Pertwee RG. 2007. Cannabidiol displays unexpectedly high potency as an antagonist of $\mathrm{CB} 1$ and $\mathrm{CB} 2$ receptor agonists in vitro. $\mathrm{Br} J$ Pharmacol 150: 613-623.

Turner BJ, Talbot K. 2008. Transgenics, toxicity and therapeutics in rodent models of mutant SOD1-mediated familial ALS. Prog Neurobiol 85: 94-134.

Wall ME, Brine DR, Perez-Reyes M. 1976. Metabolism of cannabinoids in Man. In The Pharmacology of Marijuana, Brande MC, Szara S (eds). Raven Press: New York, 93-116.

Weydt P, Hong S, Witting A, Möller T, Stella N, Kliot M. 2005 Cannabinol delays symptom onset in SOD1 (G93A) transgenic mice without affecting survival. Amyotroph Lateral Scler Other Motor Neuron Disord 6: 182-184.

Zuardi AW, Cosme RA, Graeff FG, Guimaraes FS. 1993. Effects of ipsapirone and cannabidiol on human experimental anxiety. J Psychopharmacol 7: 82-88.

Zuardi AW, Hallak JE, Dursun SM et al. 2006. Cannabidiol monotherapy for treatment-resistant schizophrenia. $J$ Psychopharmacol 20: 683-686.

Zuardi AW, Morais SL, Guimaraes FS, Mechoulam R. 1995. Antipsychotic effect of cannabidiol. J Clin Psychiatry 56: 485-486.

Zuardi AW, Rodrigues JA, Cunha JM. 1991. Effects of cannabidiol in animal models predictive of antipsychotic activity. Psychopharmacology (Berl) 104: 260-264.

Zuardi AW, Shirakawa I, Finkelfarb E, Karniol IG. 1982. Action of cannabidiol on the anxiety and other effects produced by delta-9-THC in normal subjects. Psychopharmacology 76: $245-250$ 